\title{
The Rashba-effect at metallic surfaces
}

\author{
G. Bihlmayer ${ }^{\text {a,1 }}$, Yu. M. Koroteev ${ }^{\text {b,c }}$, P. M. Echenique ${ }^{c, d}$, E. V. Chulkov ${ }^{c, d}$, and S. Blügel ${ }^{\text {a }}$ \\ a Institut für Festkörperforschung, Forschungszentrum Jülich, D-52425 Jülich, Germany \\ ${ }^{\mathrm{b}}$ Institute of Strength Physics and Materials Science, Russian Academy of Sciences, 634021, Tomsk, Russia \\ ${ }^{\mathrm{c}}$ Donostia International Physics Center 20018 San Sebastian/Donostia, Basque Country, Spain \\ d Departamento de Fsica de Materiales and Centro Mixto CSIC-UPV/EHU, Facultad de Ciencias Qumicas, UPV/EHU, \\ Apdo.1072, 20080 San Sebastian/Donostia, Basque Country, Spain
}

\begin{abstract}
Surface states, that show a k-dependent splitting resulting from spin-orbit coupling, show wide similarities to a two-dimensional electron gas in semiconductor heterostructures, where the Rashba effect lifts the spin-degeneracy of the bands and allows spin-manipulation by an electric field. We discuss the conditions for such a Rashba-effect at metallic surfaces by comparing ab initio calculations for $\mathrm{Au}(111), \mathrm{Ag}(111)$, and $\mathrm{Lu}(0001)$ and investigate the influence of electric and magnetic (exchange) fields on these surface states.
\end{abstract}

Key words: surface states, spin-orbit coupling, Rashba-effect

PACS: 71.70.Ej, 72.25.-b, 73.20.-r

\section{Spin-orbit splitting of surface states}

The discovery of the spin-orbit splitting on a $\mathrm{Au}(111)$ surface by LaShell et al. [1] using photoelectron spectroscopy (PES) provided a direct view on an electronic state, where the spin-degeneracy was lifted solely by spin-orbit coupling. The analogy between a two-dimensional electron gas (2DEG), as found in semiconductor heterostructures, and these surface states was quickly established and therefore it became common to refer to this effect also as Rashba-splitting. Here, the motion of an electron in the plane of the $2 \mathrm{DEG}$

\footnotetext{
1 Corresponding author: Tel.: +49-2461-61-4677 Fax: +492461-61-2850

http://www.fz-juelich.de/iff/staff/Bihlmayer_G

Email: G.Bihlmayer@fz-juelich.de (G. Bihlmayer)
}

(characterized by its Bloch vector $\mathbf{k}_{\|}$) through a perpendicular electric field, $E \mathbf{e}_{z}$, results in a magnetic field in the rest frame of the electron, that couples to the spin, s, of the particle as:

$H_{\mathrm{R}}=\alpha_{\mathrm{R}}\left(\mathbf{e}_{z} \times \mathbf{k}_{\|}\right) \cdot \mathbf{s}$.

Already in the paper by LaShell and coworkers [1] it was noticed, that the electric field could not be naively identified with the gradient of the surface potential. Peterson and Hedegård [2] developed a simple tight-binding model that showed that it is possible to write the Rashba parameter, $\alpha_{\mathrm{R}}$, as a product of the nuclear number $Z$ of the surface atom and a parameter describing the asymmetry of the wavefunction, $\gamma$. This asymmetry is then (also) a measure of the potential gradient at the surface and vanishes in the bulk of centro-symmetric crys- 
tals.

Although the comparison with semiconductor heterostructures has to be done with care, the bandstructures and Fermi surfaces of $\mathrm{Au}(111)$ and related surfaces [3] bear clear similarities between surface states and 2DEG's discussed in mesoscopic systems [4]. Of course, this splitting of the surface state is an effect of spin-orbit coupling and can, therefore, also be classified as "spin-orbit splitting". But to clearly distinguish this effect from other spin-orbit splitting, that do not lift the spindegeneracy of the bands, we classify this effect as Rashba effect and Rashba splitting in the following. This seems to be justified, since the symmetry requirements for this effect to appear are the same as for the "classical" Rashba effect in 2DEG's.

Recently, on other surfaces (e.g. of the semimetal $\mathrm{Bi}[5]$ ) completely different Fermi surface topologies have been found to be caused by the Rashba effect and on magnetic surfaces, like Gd(0001), interesting combinations of Rashbaand exchange splittings can be observed [6]. This opens up completely new possibilities, provided that it is somehow possible to manipulate the Rashba parameter (e.g. by an electric field) and to selectively address the surface states and separate its response from the remaining bulk-states. Especially, the semi-metallic $\mathrm{Bi}$ seems to offer good chances to overcome the second problem.

In this contribution we will first discuss the origin of the spin-orbit splitting, i.e. what characterizes a surface state and its surrounding potential so that a significant Rashba splitting can be observed. As well-known examples we will investigate $\mathrm{Au}(111)$ and $\mathrm{Ag}(111)$ and discuss why on the latter surface the splitting is more than one order of magnitude smaller. We will compare the spinorbit splitting of the $s p$-surface state on $\mathrm{Au}(111)$ and $\operatorname{Ag}(111)$ with that of $d$-type surface states on $\mathrm{Lu}(0001)$ and investigate how the Rashba constant can be manipulated in the case of a metallic surface by an external electric field.

All calculations shown here are based on density functional theory in the local density approximation and have been performed using the full-potential linearized augmented planewave method [7] as implemented in the FLEURcode [8]. Spin-orbit coupling (SOC) was included self-consistently as described in Ref.[9]. The surfaces were simulated by thick films (23 layers for $\mathrm{Au}$ and $\mathrm{Ag}, 10$ layers for $\mathrm{Lu}$ ) embedded in vacuum. The muffin-tin radii were chosen 2.5 a.u. for the noble metals and 2.8 a.u. the lanthanides. The correlation of the $4 f$-electrons of Lu was described by the LDA $+\mathrm{U}$ method [10]. The electric field was modeled as described in Ref. [11].

\section{Origin of the (Rashba) spin-orbit splitting}

In order to shed more light on the model described by Eq. 1, we calculated the splitting of surface states on different metals, i.e. on the (111) surfaces of noble metals and (0001) surfaces of lanthanides. Using density functional theory one can separate the Hamiltonian into two parts: The first one includes all scalar relativistic effects (Darwin and Mass enhancement to all orders in the speed of light) and the second one is a spin-orbit coupling (SOC) term. The latter is only important (see arguments below) in the vicinity of the nuclei and is therefore applied only in a sphere around the atom. These muffin-tin spheres are non-touching and since the potential is considered to be spherically symmetric near the nucleus - only the $l=0$ term of an expansion of the potential in spherical harmonics is taken into account [9]. In this approximation the spin-orbit coupling term takes the form

$H_{\mathrm{SO}}=\frac{1}{4 m^{2} c^{2}} \frac{1}{r} \frac{\partial V}{\partial r}(\mathbf{r} \times \mathbf{p}) \cdot \underline{\boldsymbol{\sigma}}$

where $V$ is the spherically symmetric potential, $\mathbf{p}$ is the momentum operator and $\underline{\boldsymbol{\sigma}}=\left(\underline{\sigma}_{x}, \underline{\sigma}_{y}, \underline{\sigma}_{z}\right)$ are the Pauli matrices. Although this formulation does not contain explicitly an electric field as presented in Eq. 1, calculations with this Hamiltonian reproduce the experimental Rashba splitting of the $\mathrm{Au}(111)$ surface state. Also in the case of $\operatorname{Gd}(0001)$, the agreement with experimental data was found to be quite good [6].

In the calculations we have now the possibility to resolve the contribution to the splitting of the surface state layer by layer, e.g. we can include the SO-Hamiltonian (Eq. 2) only in the surface (first subsurface,..) layer atoms and determine then 


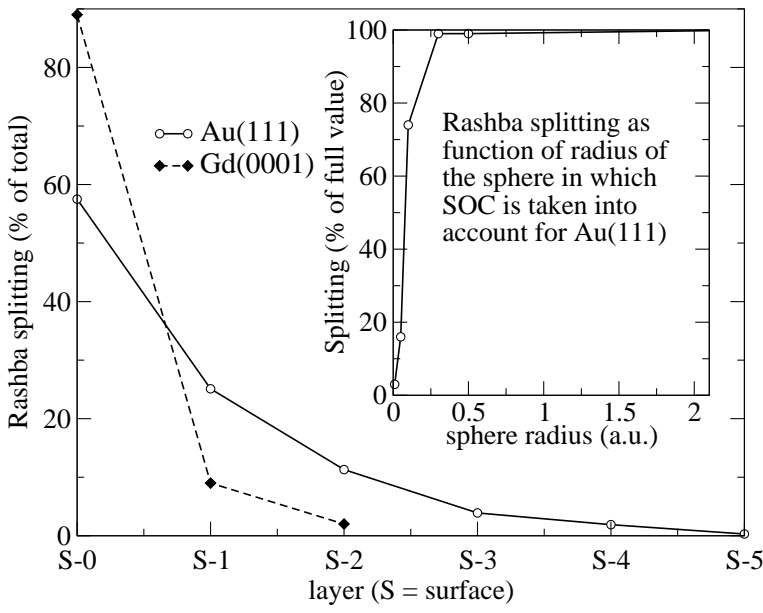

Fig. 1. Contribution to the Rashba splitting in $\mathrm{Au}(111)$ and $\operatorname{Gd}(0001)$ arising from the individual layers of a film. The surface layer is denoted by $\mathrm{S}$, deeper layers by $\mathrm{S}-1, \mathrm{~S}-2 \ldots$. Inset: contribution to the Rashba splitting coming from a sphere around the surface atom of $\mathrm{Au}(111)$ as function of the sphere radius.

the splitting in a single iteration, using the selfconsistent potential from the previous calculation when SOC was included in all atoms. In this way we arrive at a layer decomposition of the Rashba splitting as shown in Fig. 1. We see that the $\mathrm{Au}(111)$ surface states extend considerably into the bulk, in agreement with other theoretical data [12]. Note, that more than $40 \%$ of the total splitting actually comes from the subsurface layers, where the screening of the potential gradient at the surface should already be very efficient. This supports the picture that not the asymmetry of the surface potential, but the lack of z-reflection symmetry of a surface state wavefunction that is actually determining the size of the splitting.

When we vary the radius of the sphere, where the SO-Hamiltonian is taken into account, we can further refine our spatial resolution: applying a similar procedure as for the layer-decomposition of the Rashba splitting, we find that more than $90 \%$ of the contribution to the splitting comes from a region defined by a sphere of 0.25 a.u. around the nucleus. This is understandable, since the weighted potential gradient $\frac{1}{r} \frac{\partial V}{\partial r}$ is largest close to the nucleus. In this region the potential is almost perfectly radially symmetric, which explains the success of calculations with a radial symmetric potential in Eq. 2 .
Up to now, most investigations of the surface state splitting have concentrated on $\mathrm{Au}(111)$. On $\mathrm{Ag}(111)$, in contrast, ab initio calculations predict that the splitting is smaller by a factor 20 and, therefore, very difficult to observe experimentally [13]. Reinert [3] discussed this difference in terms of the potential gradient perpendicular to the surface and the amount of $p$-character in a $s p$ surface state. But actually none of these factors is sufficient to explain the large difference observed between the Ag and the Au surface state splitting. In the view of our discussion above, it is the gradient of the surface state wavefunction decaying into the bulk near the position of the nucleus that determines the size of the Rashba-splitting. Close to the nucleus, where wavefunctions are well expanded in spherical harmonics, for a surface state with predominantly $l$-character (e.g. $p$-character), such a gradient is realized by the formation of hybrid states with orbitals of $l$ - and $l \pm 1$-character. Thus, the ratio of $l$ - to $l \pm 1$-character is a measure of an effective gradient, analogous to the asymmetry parameter $\gamma$ in the model of Petersen and Hedegård [2]. In other words, a surface state of purely $p$ - or $d$-character shows no Rashba-splitting at all. Only the admixture of $d$-character to a $p$-type surface state allows the description of a decaying state and thus for a k-dependent splitting of this state that also depends on the nuclear number $Z$. Only when both, the expectation value of the weighted potential gradient $\frac{1}{r} \frac{\partial V}{\partial r}$ and the wavefunction gradient (or equivalently momentum) $\mathbf{p} \psi$ are large, a sizable splitting can be expected. (Of course the potential gradient perpendicular to the surface also modifies the wavefunction gradient and in this way enters the model. As we will see below, it is via this mechanism also possible to manipulate the Rashba-splitting with an electric field.)

Comparing now the $p: d$-ratio of the $\mathrm{Ag}$ and $\mathrm{Au}(111)$ surface states, we find that in the Ag surface atom it is rather large (9.5), while it is much smaller for $\mathrm{Au}$ (3.3). Also the ratio of $p: s$ character is larger in $\mathrm{Ag}$ than in $\mathrm{Au}$, indicating that the $p$-character of the Ag surface state is much more pronounced that in $\mathrm{Au}$ [14].

To illustrate these arguments further, we performed $a b$ initio calculations of the $\mathrm{Lu}(0001)$ surface. Like all lanthanide surfaces, this surface sup- 

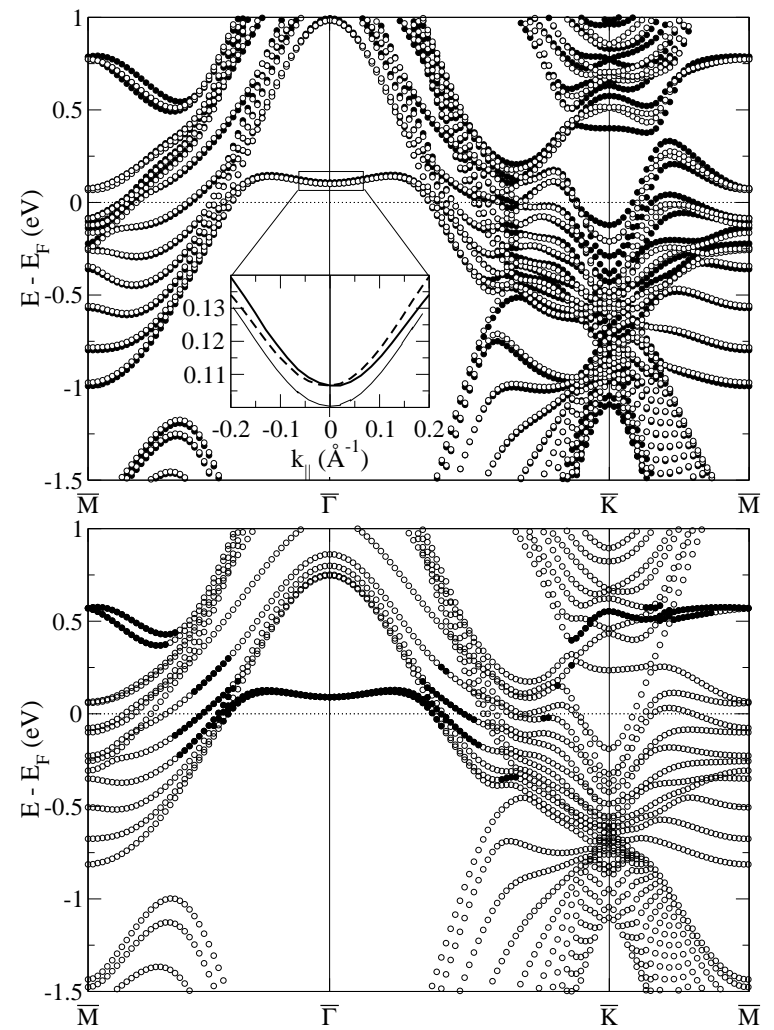

Fig. 2. Bandstructures of a relaxed 12 layer $\mathrm{Lu}(0001)$ film calculated in LDA (top) and GGA (bottom) with inclusion of a Hubbard U. The top panel shows a comparison of a calculation without (empty circles) and with (filled circles) spin-orbit coupling (SOC). In the inset, the surface state around $\Gamma$ is shown, calculated with and without SOC represented by thick and thin lines, respectively. For the GGA result (bottom), surface states are marked as filled circles, all other states are indicated by open circles.

ports a surface state near the Fermi level $\left(E_{F}\right)$, that is located in a large projected bulk-bandgap centered around the $\bar{\Gamma}$-point (Fig. 2, bottom). Lu has a rather large nuclear number $(Z=71)$, nevertheless in a comparison with bandstructures calculated with and without spin-orbit coupling the Rashba-splitting is hardly visible (Fig. 2,top). This is a consequence of the very small dispersion of the band, but as shown in an inset of Fig. 2 (top), there is actually a splitting of $0.022 \AA^{-1}$, very similar to the value in $\mathrm{Au}(111)$ which is found to be $0.025 \AA^{-1}$ both experimentally and in theory [13]. The $\mathrm{Lu}(0001)$ surface state is of $d$-type character and decays rather rapidly from the surface into the bulk, similar to what is also reflected in the fast decay of the layer resolved contribution to the Rashba-splitting in Fig. 1 for $\operatorname{Gd}(0001)$. In this case the ratio of $d: p$-character is 2.7 , so also this surface state has significant $p$ admixture.

Furthermore, a closer inspection of the bandstructure near the $\overline{\mathrm{M}}$-point shows that, for a surface state located there at about $0.78 \mathrm{eV}$ above $E_{F}$, the Rashba splitting is much larger and amounts to $0.085 \AA^{-1}$. Also the effective masses of the surface states at the $\bar{\Gamma}$ - and at the $\overline{\mathrm{M}}$-point differ by almost a factor of three: while the state at the center of the Brillouin-zone (BZ) has predominantly $d_{z^{2}}$ character with $27 \% p_{z}$-admixture, the state at the BZ boundary is mainly of $d_{x z}$-type with significantly more $p_{x}$-character (in this case $d: p$ is 1.7 ). This difference is also illustrated in the charge density plots of the surface states as shown in Fig. 3 (a) and (b). The increased $p$-character also enhances the Rashba splitting, since a $p$-type wavefunction is located much closer to the nucleus than a $d$-type wavefunction. So we see that on the same surface we can observe two surface states with completely different Rashba splitting as a consequence of the different orbital character.

\section{Influence of electric fields}

Due to the presence of a surface dipole, at any surface there is a natural electric field directed perpendicular to the surface that is proportional to the workfunction. An additional electric field applied perpendicular to the surface plane will lead to accumulation or depletion of charge near the surface naturally change the wavefunction of the surface state. If this change results in an increased (or decreased) asymmetry of the wavefunction near the nucleus, this will also influence the Rashba splitting of the surface state. To see how much the splitting can be influenced by the electric field, we included an external electric field in our calculations of $\mathrm{Lu}(0001)$.

From Fig.3 we see, that an applied (negative) electric field has the effect to deplete the surface state at the surface atom and to push charge density into the vacuum. Interestingly, the character 


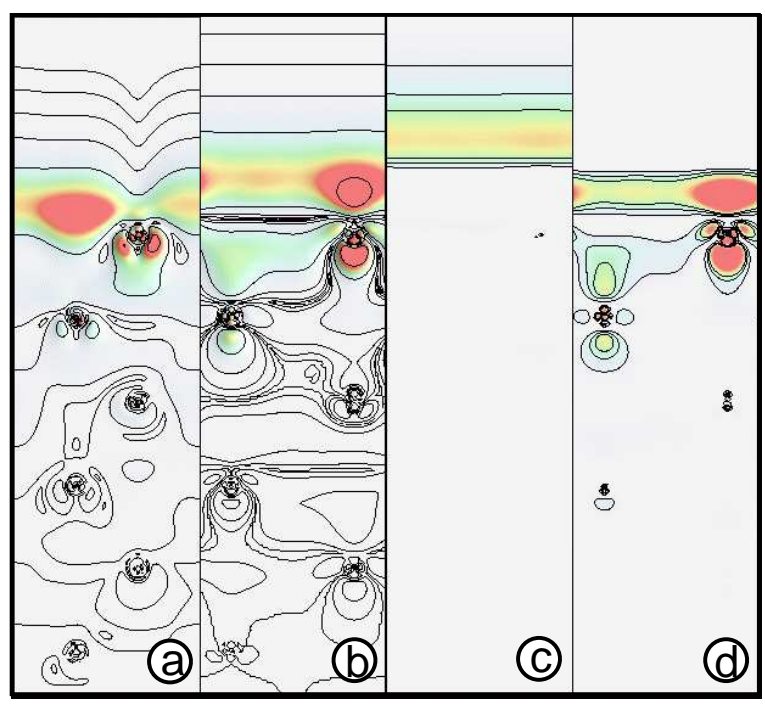

Fig. 3. (a) Charge density of the surface state of $\mathrm{Lu}(0001)$ at the $\overline{\mathrm{M}}$-point (a) and $\bar{\Gamma}$-point (b) (contour lines in a logarithmic mesh); Right: charge density increase (c) and decrease (d) of the surface state at $\bar{\Gamma}$, when an electric field of $-0.46 \mathrm{~V} / \AA$ is applied.

of the surface state does not change significantly and the ratio $d: p$ is again about 2.7 , but the total charge in the muffin-tin decreases by $7 \%$ when a field of $-0.46 \mathrm{~V} / \AA$ is applied. As a consequence, the Rashba splitting of $\mathrm{Lu}(0001)$ decreases by about $10 \%$ to $0.020 \AA^{-1}$. Although this effect seems to be small and the applied electric field large, we want to emphasize that - compared to the effect in semiconductor heterostructures - the Rashba effect is already large, and also its variation by $10 \%$ is therefore substantial.

Additionally, the electric field shifts the surface state at $\bar{\Gamma}$ down in energy, so that it moves closer to the Fermi-level. The surface state at the $\overline{\mathrm{M}}$-point, in contrast, does not change its position relative to the Fermi level and also its Rashba splitting is almost unaffected by an external electric field. The question, whether it is possible to manipulate the size of the splitting of a surface state via an electric field, depends sensitively on the character of the state itself.

\section{Summary}

We investigated the conditions that lead to a $\mathbf{k}$-dependent splitting of the surface states in $\mathrm{Au}(111), \mathrm{Ag}(111)$ and $\mathrm{Lu}(0001)$ caused by spinorbit coupling. The asymmetry of the wavefunction near the nucleus, characterized by the ratios of different $l$-components of an expansion of the wavefunction of the surface state in spherical harmonics, provides a good measure for the strength of the splitting. Furthermore, an external electric field allows to manipulate the size of the splitting in certain cases, underlining the similarity with the Rashba effect in semiconductor heterostructures.

\section{References}

[1] S. LaShell, B. A. McDougall, and E. Jensen, Phys. Rev. Lett. 77, 3419 (1996).

[2] L. Petersen and P. Hedegård, Surf. Sci. 459, 49 (2000).

[3] F. Reinert, J. Phys.: Condens. Matter 15, S693 (2003).

[4] L. W. Molenkamp, G. Schmidt, and G. E. W. Bauer, Phys. Rev. B 64, 121202 (R) (2001).

[5] Yu. M. Koroteev, G. Bihlmayer, J. E. Gayone, E. V. Chulkov, S. Blügel, P. M. Echenique, and Ph. Hofmann, Phys. Rev. Lett. 93, 046403 (2004).

[6] O. Krupin, G. Bihlmayer, K. Starke, S. Gorovikov, J. E. Prieto, K. Döbrich, S. Blügel, and G. Kaindl Phys. Rev. B 71, 201403 (R) (2005).

[7] E. Wimmer, H. Krakauer, M. Weinert, and A. Freeman, Phys. Rev. B 24, 864 (1981).

[8] For program description see http://www.flapw.de.

[9] C. Li, A. J. Freeman, H. J. F. Jansen, and C. L. Fu, Phys. Rev. B 49, 5433 (1990).

[10] V. I. Anisimov, F. Aryasetiawan, and A. I. Lichtenstein, J. Phys.:Condens. Matter 9767 (1996).

[11] S. Heinze, X. Nie, S. Blügel, and M. Weinert, Chem. Phys. Lett. 315, 167 (1999).

[12] J. Henk, A. Ernst, and P. Bruno, Phys. Rev. B 68, 165416 (2003).

[13] G. Nicolay, F. Reinert, S. Hüfner, and P. Blaha, Phys. Rev. B 65, 033407 (2001).

[14] The decomposition in l-type characters depends of course also on the radius of the sphere, in which the $(l, m)$-decomposition of the wavefunction is performed. 